\title{
Density functional study of gold atoms and clusters on a graphite (0001) surface with defects
}

\author{
Jaakko Akola ${ }^{1,2}$ and Hannu Häkkinen ${ }^{2}$ \\ ${ }^{1}$ Institut für Festkörperforschung, Forschungszentrum Jülich, D-52425 Jülich, Germany \\ ${ }^{2}$ Nanoscience Center, Department of Physics, P.O. Box 35, FI-40014 University of Jyväskylä, Finland
}

(Received 2 June 2006; published 6 October 2006)

\begin{abstract}
Adsorption of gold atoms and clusters $(N=6)$ on a graphite (0001) surface with defects has been studied using density functional theory. In addition to perfect graphite (0001), three types of surface defects have been considered: a surface vacancy (hole), a pyridinelike defect comprising three grouped nitrogen atoms, and a substitutional doping by $\mathrm{N}$ or $\mathrm{B}$. Results for $\mathrm{Au}$ and $\mathrm{Au}_{6}$ indicate that the surface vacancy can form chemical bonds with $\mathrm{Au}$ as the three nearby carbons align their dangling bonds towards the gold particle (binding energy 2.4-2.6 eV). A similar chemically saturated holelike construction with three pyridinic $\mathrm{N}$ atoms results in a significant polarization interaction $(1.1-1.2 \mathrm{eV})$, whereas the binding with the perfect graphite surface is weak $(\sim 0.3 \mathrm{eV})$. The corresponding energies for the $\mathrm{B} / \mathrm{N}$ substituted surface are $0.8-1.2 \mathrm{eV}$ (B) and $0.2-0.6 \mathrm{eV}$ $(\mathrm{N})$, and the $\mathrm{N}$ impurity donates charge to $\mathrm{Au} / \mathrm{Au}_{6}$. Several $\mathrm{Au}_{6}$ isomers have been tested in different orientations on substrate, and the triangular gas-phase geometry $\left(D_{3 h}\right)$ standing on its apex is a low-energy configuration ( $\mathrm{N}$ substitution is an exception). In general, coordination through corner atoms is energetically favorable. For the surface vacancy, the presence of gold particles leads to a significant surface reconstruction, whereas the pyridinelike defect appears rigid. There is no significant charge transfer, and the net charge on $\mathrm{Au}_{6}$ ranges between $-0.2 e$ and $0.1 e$.
\end{abstract}

DOI: 10.1103/PhysRevB.74.165404

PACS number(s): 68.43.Bc, 36.40.-c, 81.05.Uw, 61.72.Ji

\section{INTRODUCTION}

Much recent effort in cluster science has focused on phenomena associated with the interactions of nanoclusters with solid substrates (for reviews see, e.g., Refs. 1-5). Energetic cluster beams can be used, for example, to modify the structural, electronic, and magnetic properties of the substrate, with the aim of growing thin films with an internal structure that is controllable on the nanometer scale. ${ }^{4,6}$ Moreover, one may be interested in the physical and chemical properties of the as-deposited, isolated clusters themselves. ${ }^{5,7-9}$ Small gold clusters exhibit distinct electronic, optical, and chemical properties that are extremely sensitive to the cluster size and the charge state ${ }^{10-12}$ and provide attractive nanoscale building blocks for catalysis, labeling, photonics, and sensing. Engineering and tuning the cluster properties for potential applications in these areas require fundamental knowledge of the interaction between a cluster and its environment.

The atomic smoothness and chemical inertness of highly ordered pyrolytic graphite (HOPG) means that it is often used to support metal nanoparticles. However, noble metal atoms are weakly adsorbed on HOPG and show high lateral mobility (diffusion). This is reflected in the formation of dendritic Au particles on $\mathrm{HOPG},{ }^{13-15}$ and in the spontaneous nucleation of bare Au clusters on single-walled carbon nanotubes (SWNTs). ${ }^{16,17}$ The gold-graphite interface has previously been studied theoretically in the context of adatom and cluster diffusion, ${ }^{18-22}$ but less attention has been paid to the "functionalization" of the interface by chemical modifications and to the elucidation of their effects on the properties of gold adsorbates. It should be noted that the basic information extracted from studies of gold/graphite interface may also be applied to the functionalization of large-diameter (low-curvature) carbon nanotube (CNT) side walls. ${ }^{23}$

An effective method for preventing diffusion after deposition is provided if clusters impinge on the substrate with a high kinetic energy, leading to a local distortion of the hexagonal graphite lattice. ${ }^{24,25}$ Furthermore, defects introduce pinning centers for metal particles that can promote cluster and film growth, and one monolayer deep nanometer-sized pits have been used to trap $\mathrm{Ag}$ and $\mathrm{Au}$ clusters on HOPG. ${ }^{26-28}$ In the context of SWNT electronic devices, it has been shown that Ni clusters can be used to identify and count chemically active atomic defects on HOPG and CNTs. ${ }^{29}$ As recently confirmed in situ, ${ }^{30}$ typical atomic defects obtained by knock-on atom displacements include pentagon-heptagon pairs, mono-vacancies and multivacancies (e.g., 5-8-5 divacancy), adatoms, and interstitials. Another class of atomic defects on graphene layers are obtained by $\mathrm{B} / \mathrm{N}$ doping, where the impurity atom introduces either an additional conduction electron $(\mathrm{N})$ or a hole in the valence band (B). In addition to single carbon atom replacements, holelike constructions of three $\mathrm{N}$ atoms (pyridinelike defects) have been observed in pyrolysis synthesized N-doped CNTs, and they are responsible for the enhanced metallic character of these nanostructures. ${ }^{31}$

We report here a density functional theory (DFT) based investigation of the adsorption of gold adatoms and $\mathrm{Au}_{6}$ clusters on a graphite (0001) surface with defects. $\mathrm{Au}_{6}$ is chosen as a representative of small gold clusters on HOPG because of the chemical stability of its gas phase geometry [large energy gap between the highest occupied molecular orbital (HOMO) and lowest unoccupied molecular orbital (LUMO)], and according to our knowledge this cluster size has not been studied on a graphite support at this level of theory. The substrate is modeled as a periodic slab of two graphene layers, and its electronic band structure is taken into account by a k-point mesh in the reciprocal space. Although the interaction between a gold adatom and an ideal HOPG surface is weak $(\sim 0.3 \mathrm{eV})$, it can be modified easily by defects, and we have considered an unsaturated carbon 

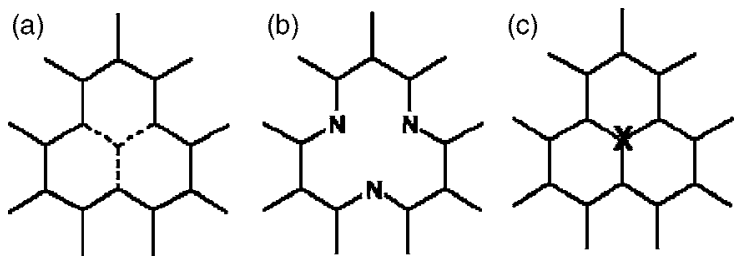

FIG. 1. Three types of surface defects on graphite: (a) vacancy, (b) pyridinelike defect of three $\mathrm{N}$ atoms, and (c) substitution $(X$ $=\mathrm{B} / \mathrm{N})$.

vacancy, substitutional $\mathrm{N}$ and $\mathrm{B}$ atoms, and a pyridinelike defect where the vacancy is chemically stabilized by three $\mathrm{N}$ atoms (see Fig. 1). All these defects are relevant to the functionalization of HOPG or CNT side walls, and they cover a diverse range of gold-support interactions with the adsorption energies varying from 0.2 to $2.6 \mathrm{eV}$. Analysis of the electron localization function ${ }^{32}$ (ELF) and the electron density redistribution upon bonding reveals that the adsorption has a mixed metal-covalent character for a vacancy, and the pyridinelike defect and B substitution exhibit a visible polarization both in the substrate and adsorbate. A clear case where charge transfer to gold takes place is provided by the substitutional $\mathrm{N}$ atom ("graphitic nitrogen"), which acts as a strong electron donor to the Au $6 s$ orbital. The energetically favorable isomers of adsorbed $\mathrm{Au}_{6}$ are usually those with a single-atom Au contact to the support.

\section{SIMULATION METHODS}

The calculations have been performed with the CarParrinello molecular dynamics package (CPMD) ${ }^{33}$ which is based on the density functional theory of electronic structure. The generalized gradient approximation of Perdew, Burke, and Ernzerhof $(\mathrm{PBE})^{34}$ is adopted for the exchangecorrelation energy functional, and the electron-ion interaction is described by ionic pseudopotentials with the nonlocal, norm-conserving, and separable form suggested by Troullier and Martins. ${ }^{35}$ For Au atoms, the $5 d$ electrons are explicitly included in the valence electrons, and the scalar-relativistic pseudopotential contains nonlinear core corrections. The program employs periodic boundary conditions, and the plane wave basis set has a kinetic energy cutoff of $70 \mathrm{Ry}$. The electronic Hamiltonian is rediagonalized after each geometry optimization step, and a finite temperature functional ( $T$ $=1000 \mathrm{~K})$ by Alavi et al. ${ }^{36}$ is used for the Kohn-Sham (KS) orbital occupancies due to the semimetallic nature of graphite (small band gap).

Our model of the periodic substrate of Bernal graphite comprises two graphene layers (stacking $A B$ ) in a hexagonal supercell of $14.77 \times 14.77 \times 15.36 \AA^{3}$ (144 C atoms). The perpendicular distance between the periodic replicas of $(12 \AA)$ enables vertical orientations of $\mathrm{Au}_{6}$ clusters without a significant interaction with the neighboring slab, and the choice of unit cell keeps the adsorbates around $10 \AA$ apart. Earlier results for the Na adsorption on HOPG (Ref. 37) indicated that three graphene layers are needed to converge the adsorption energy and distance. However, the nature of Au-HOPG interaction is different (especially in the case of
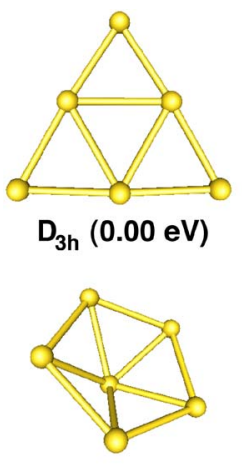

$\mathrm{C}_{5 \mathrm{v}}(0.86 \mathrm{eV})$

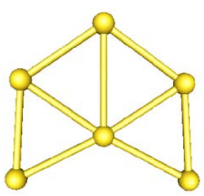

$\mathrm{C}_{2 v}(\mathrm{~m})$

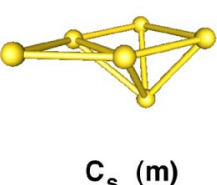

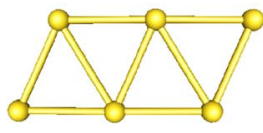

$D_{2 h}(1.21 \mathrm{eV})$

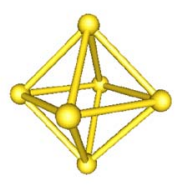

$O_{h}(1.78 \mathrm{eV})$
FIG. 2. (Color online) $\mathrm{Au}_{6}$ isomers and their corresponding total energies with respect to the lowest energy isomer $\left(D_{3 h}\right)$. Isomers $C_{2 v}$ and $C_{s}$ are metastable (denoted by " $m$ ") in the gas phase.

defects), and our test calculations for a single Au atom on a surface vacancy showed little difference between the results for two and three graphene layers. ${ }^{38}$ The spacing between the layers is fixed to the experimental value $(3.35 \AA)$, since the PBE functional does not describe weak dispersion forces well. ${ }^{39} \mathrm{On}$ the other hand, the adsorption energy of Au on HOPG agrees well with the experimental information (see below). Tests for different numbers of $\mathbf{k}$ points indicate that the $\Gamma$-point approximation is adequate for geometry optimization (the corresponding band gap is zero for the unit cell chosen), whereas the adsorption energies and electronic band structure are calculated with a $5 \times 5 \times 1$ Monkhorst-Pack k-point mesh. ${ }^{40}$

The effect of surface relaxation has been incorporated for defect-free graphite by releasing constraints on $37 \mathrm{C}$ atoms beneath the Au particle, and these atoms can also relax for the substrates with surface defects. The fixed graphene layer spacing causes an effective repulsion, which is evident as a slight bending of the constraint-free region (maximum vertical displacement $0.09 \AA$ ). The ionic positions are optimized using a quasi-Newton approach (BFGS method) ${ }^{41}$ until all the components of nuclear gradients are below 3 $\times 10^{-4}$ a.u., and the change of the total energy between two successive optimization steps is less than $1 \times 10^{-5} \mathrm{H}$.

\section{RESULTS}

Previous studies of free small Au clusters ${ }^{10-12}$ have shown that planar geometries are favored energetically due to the relativistic effects that increase $s d$ hybridization. Our results for selected $\mathrm{Au}_{6}$ geometries and the corresponding total energies are shown in Fig. 2, confirming that the $\mathrm{Au}_{6}$ ground state is planar with a $D_{3 h}$ symmetry. The corresponding binding energy is $1.90 \mathrm{eV}$ per atom and the HOMO-LUMO gap is $2.07 \mathrm{eV}$. Two other planar geometries are presented, and the $C_{2 v}$ geometry is metastable $(0.5 \mathrm{eV}$ above, fixed geometry) relaxing back to the ground state, and $D_{2 h}$ is considerably higher in energy. The most stable $3 \mathrm{D}$ isomer $\left(C_{5 v}\right)$ is $0.86 \mathrm{eV}$ higher than the planar ground state. The metastable $C_{s}$ isomer (1.1 eV above, fixed geometry) relaxes to the $D_{3 h}$ geometry, and the symmetric octahedron $\left(O_{h}, 1.78 \mathrm{eV}\right)$ is 
TABLE I. Au atom and $\mathrm{Au}_{6}$ cluster on graphite (0001) with and without surface defects: perfect graphite (GR2, $X=\mathrm{C}$ ), a surface vacancy (GR2-h, $X=3 \times \mathrm{C}$ ), nitrogen (GR2-N, $X=\mathrm{N}$ ) and boron substitutions (GR2-B, $X=\mathrm{B})$, and a pyridinelike defect $(\mathrm{GR} 2-\mathrm{N} 3, X=3 \times \mathrm{N}) . E_{a}$ is calculated for both the $\Gamma$ point (in parenthesis) and $5 \times 5 \times 1 \mathbf{k}$-point mesh, but the geometries are optimized using the $\Gamma$ point alone. The vertical displacements from the upper graphene plane $\left[d_{\perp}(\mathrm{Au})\right.$ and $\left.d_{\perp}(X)\right]$, the nearest-neighbor $X$-C distances within the graphene plane, and the estimated charge transfer $(\Delta q)$ are reported also. $\Delta q>0$ implies a charge transfer to gold.

\begin{tabular}{llllll}
\hline \hline & \multicolumn{1}{c}{ GR2 } & GR2-h & GR2-N & GR2-B & GR2-N3 \\
\hline Au atom: & & & & & \\
$E_{a}$ & $0.25(0.51)$ & $2.62(2.33)$ & $0.57(0.38)$ & $1.18(0.96)$ & $1.15(0.82)$ \\
$\mathrm{Au}-X$ & 2.25 & 2.09 & 3.37 & 0.20 & 2.36 \\
$d_{\perp}(\mathrm{Au})$ & 2.70 & 1.95 & 3.43 & 2.91 & 2.11 \\
$d_{\perp}(X)$ & 0.45 & 0.62 & 0.06 & 0.71 & 0.30 \\
$X-\mathrm{C}$ & 1.46 & 1.40 & 1.41 & 1.52 & 1.35 \\
$\Delta q$ & 0.14 & -0.24 & 0.41 & 0.08 & -0.10 \\
$\mathrm{Au}$ & & & & & \\
$E_{a} \mathrm{cluster}\left(D_{3 h}\right):$ & & $2.43(2.41)$ & $0.20(0.15)$ & $0.83(0.74)$ & $1.15(1.05)$ \\
$\mathrm{Au}-X$ & $0.33(0.34)$ & 2.10 & 4.12 & 2.33 & $2.29,2.49$ \\
$d_{\perp}(\mathrm{Au})$ & 2.38 & 1.97 & 3.89 & 2.87 & 2.20 \\
$d_{\perp}(X)$ & 2.61 & 0.60 & 0.11 & 0.54 & $0.26,0.39$ \\
$\Delta q$ & 0.24 & -0.06 & 0.19 & 0.13 & 0.14 \\
\hline \hline
\end{tabular}

energetically unfavorable. The bond lengths of the $D_{3 h}$ isomer are $2.64 \AA$ for the peripheral bonds and $2.79 \AA$ for the central triangle. In general, $\mathrm{Au}_{6}$ clusters exhibit bond lengths between 2.6-2.9 ̊ depending on the coordination.

Results of the Au adatom adsorption on a graphite substrate with and without atomic defects (see Fig. 1) are summarized in Table I. The adsorption energy $E_{a}$ is presented both for the $\Gamma$-point approximation and $5 \times 5 \times 1 \mathbf{k}$-point mesh, and it shows a deviation of $0.2-0.3 \mathrm{eV}$ in each case. For pristine HOPG (labeled as GR2), the $\Gamma$-point approximation overestimates adsorption, whereas the situation is reversed for the defects. The strongest binding $(2.6 \mathrm{eV})$ is observed for the vacancy (GR2-h) as the dangling bonds of three carbons interact with the adatom. Also the pyridinelike defect (GR2-N3) and boron substitution (GR2-B) show considerable binding $(1.2 \mathrm{eV})$, whereas the nitrogen doped case (GR2-N) and defect-free GR2 bind Au weakly. Our result for the Au adsorption energy on HOPG $(0.25-0.51 \mathrm{eV})$ is consistent with the experimentally (indirectly) determined value $\Delta E=E_{a}-E_{d}=0.39 \pm 0.04$ where the diffusion barrier $E_{d}$ is estimated to be small. ${ }^{13,14}$ Earlier DFT studies ${ }^{20,22}$ report diffusion barriers of $0.02-0.05 \mathrm{eV}$ and adsorption energies of $0.7-$ $0.8 \mathrm{eV}$ for a single Au on HOPG by using the local density approximation (LDA) for the exchange-correlation energy functional.

Despite the small adsorption energy of Au on HOPG, the vertical displacement of the underlying substrate atom $\left[d_{\perp}(\mathrm{C})=0.45 \AA\right]$ shows a noticeable relaxation of GR2 in Table I. Analysis of the laterally averaged charge density difference curve in Fig. 3(a) reveals that this is a polarization effect, where the accumulated net charge $(\Delta q)$ on $\mathrm{Au}$ is $0.14 e .{ }^{42}$ The corresponding LDA results by Wang et al. ${ }^{20}$ are $0.28 \AA$ for the surface relaxation $\left[d_{\perp}(\mathrm{C})\right]$ and $0.17 e$ for the accumulated charge on Au. Large structural deformations are observed also for GR2-h (dangling bonds) ${ }^{43,44}$ and GR2-B $\left(s p^{3}\right.$ hybridization), whereas the graphitic and pyridinic $\mathrm{N}$ atoms of GR2-N and GR2-N3 stay in plane. The charge density difference plots for GR2-h and GR2-N [Figs. 3(b) and 3(c)] exhibit simple patterns where Au donates charge to the vacancy $(-0.24 e)$ and accepts charge from the $\mathrm{N}$ impurity (0.41e), respectively. The corresponding contours for GR2-B and GR2-N3 [Figs. 3(d) and 3(e)] are more complicated, and the resulting net charges are close to zero.

The charge density difference of $\mathrm{Au}$ on GR2-N and GR2-B (Fig. 4) is obtained by subtracting the electron density of the separated $\mathrm{Au}$ and substrate from the density of the whole system. In the case of nitrogen doping [Fig. 4(a)], the substrate has an additional conduction electron that does not participate in $s p^{2}$ bonding. Part of the corresponding electron density is depleted (blue) from the vicinity of $\mathrm{N}$ and accumulated (red) spherically around Au (6s orbital). The charge depletion close to the Au core is due to the Coulomb selfrepulsion of the accumulated charge $(0.41 e)$. The situation is reversed for boron doping [Fig. 4(b)], where the substrate has a delocalized "hole" in its valence band. Charge is attracted from Au towards B, but it undergoes a complicated redistribution throughout the system, and the evaluation of the charge transfer becomes difficult.

In addition to a single Au atom, Table I contains information for the $\mathrm{Au}_{6}$ adsorption corresponding to a configuration where the triangular cluster $\left(D_{3 h}\right.$, Fig. 2) stands on its apex (face-down orientation for GR2-N). The adsorption energies compared with the adatom for GR2, GR2-h, and GR2-N3 are similar, and the same applies to the distances from the substrate. Furthermore, the $E_{a}$ deviation between the $\Gamma$-point approximation and the $5 \times 5 \times 1 \mathbf{k}$-point mesh is significantly 


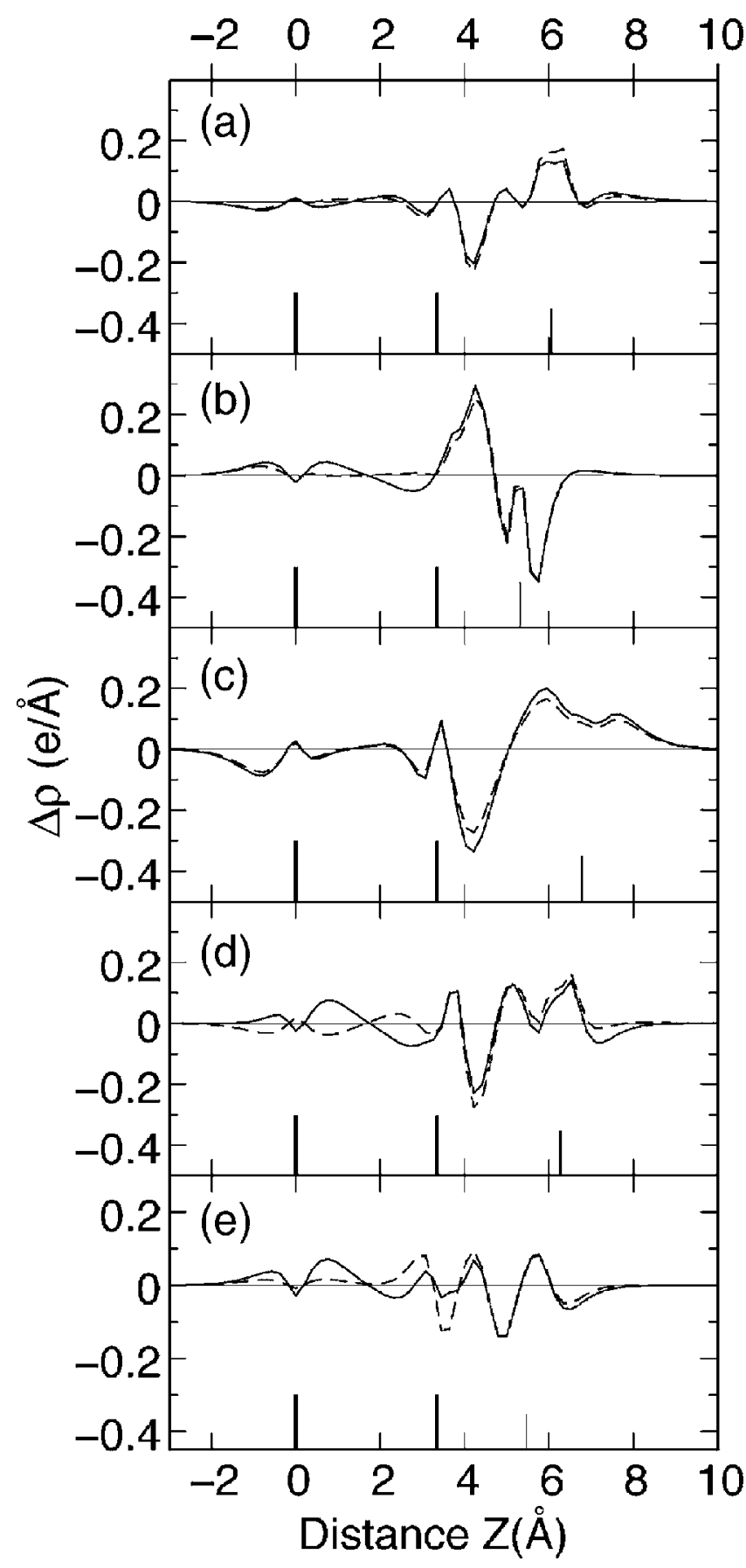

FIG. 3. Laterally averaged charge density difference $(\Delta \rho, z$ direction) of $\mathrm{Au}$ on (a) perfect graphite (GR2), (b) surface vacancy (GR2-h), (c) nitrogen substitution (GR2-N), (d) boron substitution (GR2-B), and (e) pyridinelike defect (GR2-N3). The solid and dashed lines correspond to the $5 \times 5 \times 1 \mathrm{k}$-point mesh and $\Gamma$-point approximation, respectively. The vertical bars mark the positions of graphene layers (thicker bars) and Au (thinner bar).

less in each case. The surface relaxation effect of GR2 $\left(d_{\perp}\right.$ $=0.24 \AA$ ) is smaller than for $\mathrm{Au}$ atom and is compensated by the increased Au-C distance of $2.38 \AA$. The estimated charge transfer is the same for GR2 as previously, whereas the net charge on $\mathrm{Au}_{6}$ is negligible for GR2-h, and $\mathrm{Au}_{6}$ accepts a small amount of charge $(0.14 e)$ from GR2-N3. The $\mathrm{B} / \mathrm{N}$
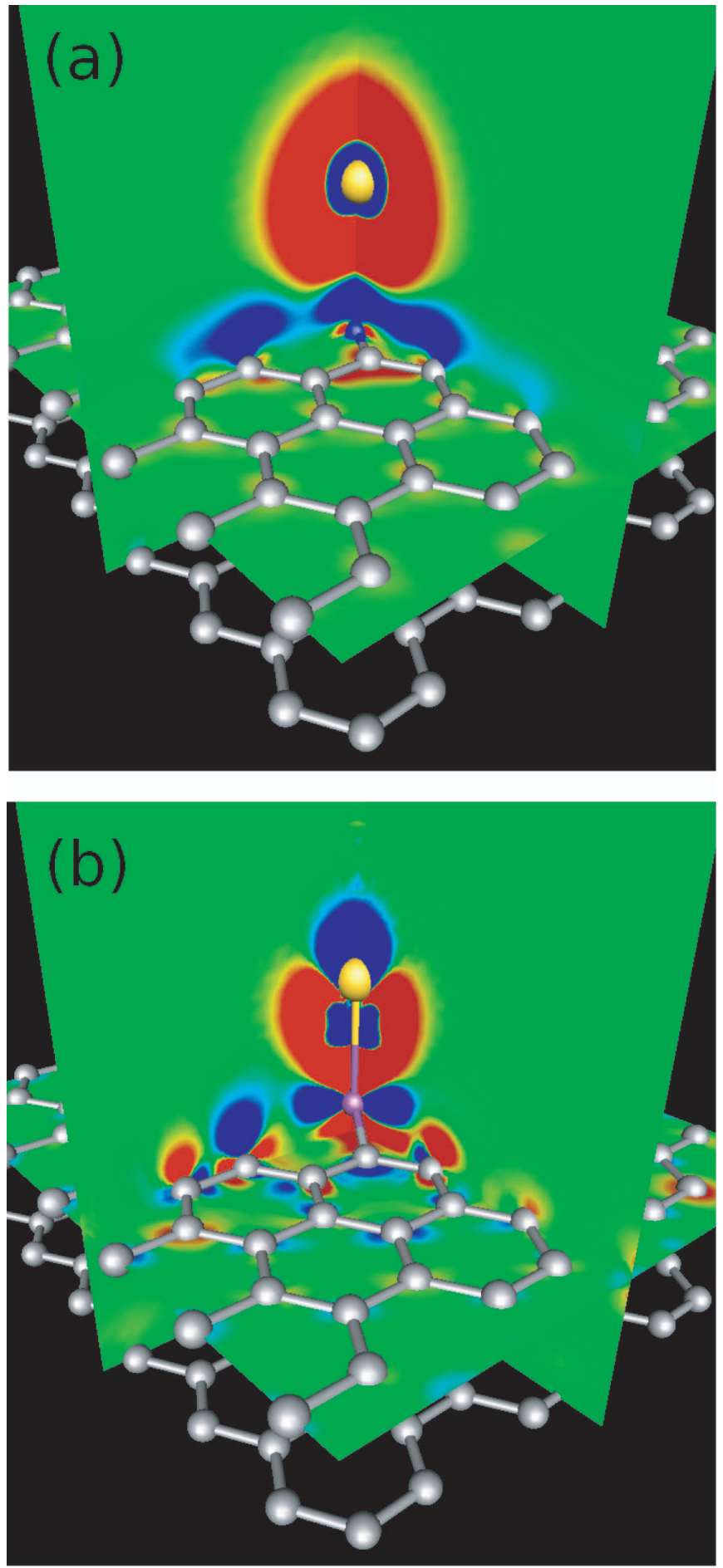

FIG. 4. (Color) Cutplane visualization of the charge density difference of $\mathrm{Au}$ on (a) nitrogen and (b) boron substitution. The red color corresponds to accumulation $\left(0.0005 e / \AA^{3}\right.$ or more $)$ and blue depletion $\left(-0.0005 e / \AA^{3}\right.$ or less). Gold, nitrogen, and boron atoms are marked by a yellow, blue, and magenta sphere, respectively.

defects interact more weakly with $\mathrm{Au}_{6}$ than with the adatom, but a noticeable binding with the cluster apex and GR2-B $(0.8 \mathrm{eV})$ remains. $\mathrm{Au}_{6}$ does not prefer the apex orientation on GR2-N and aligns parallel to the substrate (separation $4.12 \AA$ ). The corresponding adsorption energy is smaller 
TABLE II. $\mathrm{Au}_{6}$ isomers on graphite (0001) with and without surface defects: perfect graphite (GR2, $X$ $=\mathrm{C})$, a surface vacancy (GR2-h, $X=3 \times \mathrm{C})$, and a pyridinelike defect (GR2-N3, $X=3 \times \mathrm{N}) . \Delta E$ is calculated for both the $\Gamma$ point (in parenthesis) and $5 \times 5 \times 1 \mathbf{k}$-point mesh, but the geometries are optimized using the $\Gamma$ point alone.

\begin{tabular}{|c|c|c|c|c|}
\hline & $\Delta E(\mathrm{eV})$ & $\mathrm{Au}-X$ & $d_{\perp}(\mathrm{Au})(\AA)$ & $d_{\perp}(X)(\AA)$ \\
\hline \multicolumn{5}{|l|}{ GR2: } \\
\hline$D_{3 h}($ apex $)$ & $0.00(0.00)$ & 2.38 & 2.61 & 0.24 \\
\hline$D_{3 h}$ (edge) & $0.18(0.20)$ & $3.23-3.58$ & $3.28,3.35$ & 0.12 \\
\hline$D_{3 h}($ face $)$ & $0.29(0.32)$ & $3.49,3.54$ & $3.53,3.58$ & 0.06 \\
\hline$C_{2 v}$ & $0.61(0.57)$ & $2.45,2.54$ & $2.62,2.67$ & 0.22 \\
\hline$C_{5 v}(3 \mathrm{D})$ & $0.96(0.96)$ & 2.39 & 2.62 & 0.23 \\
\hline$D_{2 h}$ & $1.33(1.15)$ & 2.30 & 2.70 & 0.40 \\
\hline$O_{h}(3 \mathrm{D})$ & $1.64(1.47)$ & 2.27 & 2.68 & 0.41 \\
\hline \multicolumn{5}{|l|}{ GR2-h: } \\
\hline$D_{2 h}$ & $0.00(0.00)$ & $2.09-2.17$ & 2.09 & 1.20 \\
\hline$D_{3 h}$ (apex) & $0.08(-0.14)$ & 2.10 & 1.97 & 0.60 \\
\hline$C_{s}(3 \mathrm{D})$ & $0.27(0.15)$ & $2.06-2.11$ & 1.88 & 0.54 \\
\hline$C_{2 v}$ & $0.42(0.28)$ & 2.10 & 1.94 & 0.70 \\
\hline$O_{h}(3 \mathrm{D})$ & $0.81(0.64)$ & 2.08 & 1.90 & 0.55 \\
\hline$D_{3 h}($ face $)$ & $2.39(2.15)$ & $3.58-3.64$ & $3.69-3.74$ & 0.10 \\
\hline \multicolumn{5}{|l|}{ GR2-N3: } \\
\hline$D_{3 h}$ (apex) & $0.00(0.00)$ & $2.29,2.49$ & 2.20 & $0.26,0.39$ \\
\hline$C_{2 v}$ & $0.50(0.50)$ & $2.27,2.50$ & 2.14 & $0.14,0.47$ \\
\hline$C_{s}(3 \mathrm{D})$ & $0.72(0.86)$ & $2.32,2.43$ & 2.11 & $0.24,0.35$ \\
\hline$D_{3 h}$ (edge) & $0.80(0.73)$ & $2.82-3.37$ & $2.71-3.08$ & $-0.08,0.32$ \\
\hline$D_{2 h}$ & $0.99(1.11)$ & $2.14,2.49$ & 2.11 & $0.19,0.50$ \\
\hline$C_{5 v}(3 \mathrm{D})$ & $1.11(1.18)$ & $2.47,2.50$ & 2.34 & $0.35-0.38$ \\
\hline$D_{3 h}($ face $)$ & $1.17(1.04)$ & $3.50-3.56$ & $3.51-3.58$ & $0.02-0.05$ \\
\hline$O_{h}(3 \mathrm{D})$ & $1.62(1.74)$ & $2.36,2.41$ & 2.15 & $0.28,0.36$ \\
\hline
\end{tabular}

than for GR2, and charge has accumulated on $\mathrm{Au}_{6}(0.19 e)$.

Several $\mathrm{Au}_{6}$ isomers have been optimized in different orientations on GR2, GR2-h, and GR2-N3. Table II contains the total energies $\Delta E$ relative to the lowest energy configuration and structural parameters for selected configurations, and Fig. 5 shows two low energy configurations for each substrate. For GR2, the small adsorption energies $(<0.5 \mathrm{eV})$ do not overcome the total energy differences of free $\mathrm{Au}_{6}$ isomers, and consequently, the three $D_{3 h}$ configurations have the lowest energies. In the case of $D_{3 h}$ face-down orientation, the interaction between the cluster and substrate is negligible $\left[d_{\perp}(\mathrm{Au})=3.5 \AA\right]$. Comparison with the other isomers shows similar energy differences to the gas phase values, and the clusters bind through corner atoms to the underlying $\mathrm{C}$ atom. The first geometry that is not based on $D_{3 h}$ isomer is the $C_{2 v}$ configuration (Fig. 5) where the metastable isomer is stabilized by the substrate (two Au-C contacts). Furthermore, the $C_{5 v}$ configuration is the first containing a $3 \mathrm{D}$ isomer, but the high coordination of the corner $\mathrm{Au}$ weakens adsorption, and the energy difference with respect to the lowest energy configuration is larger than the corresponding gas phase value. The $C_{s}$ isomer (Fig. 2) is not stable on GR2, and the cluster reverts to the $D_{3 h}$ geometry. The strongest interaction be- tween $\mathrm{Au}_{6}$ and GR2 is observed for the octahedron $\left(O_{h}\right)$ despite its high total energy, and the resulting vertical adsorption energy is $0.47 \mathrm{eV}$ [Au-C distance $2.27 \AA$ and $\left.d_{\perp}(\mathrm{C})=0.41 \AA\right]$.

The vacancy in GR2-h results in three dangling bonds in the surrounding three $\mathrm{C}$ atoms that are able to bind strongly with $\mathrm{Au}_{6}$. In general, this yields a strong local deformation in the substrate and can be seen as a bulging of the contact area in Fig. 5. The low energy configurations $D_{2 h}$ and $D_{3 h}$ have similar energies, and their ordering changes as the $\Gamma$-point calculation is extended to the $5 \times 5 \times 1 \mathbf{k}$-point mesh. The high stability of the $D_{2 h}$ isomer is caused by a strong substrate deformation where one carbon points towards the cluster $\left[d_{\perp}(\mathrm{C})=1.20 \AA\right]$ binding with two Au atoms simultaneously, and the resulting total $\mathrm{Au}-\mathrm{C}$ coordination is four with a vertical adsorption energy of $3.72 \mathrm{eV}$. For $D_{3 h}$, the substrate deformation is smaller $\left[d_{\perp}(\mathrm{C})=0.60 \AA\right]$ and symmetric (Au-C coordination 3), and the Au-C bonds of $2.10 \AA$ are significantly shorter than for the defect-free GR2. Other cluster motives are closer to the lowest energy configuration, and the first 3D isomer $C_{s}$ (metastable on GR2) is only $0.27 \mathrm{eV}$ higher in total energy. The $C_{5 v}$ isomer is not stable on GR2-h, as it optimizes to $C_{s}$ due to the strong interaction 

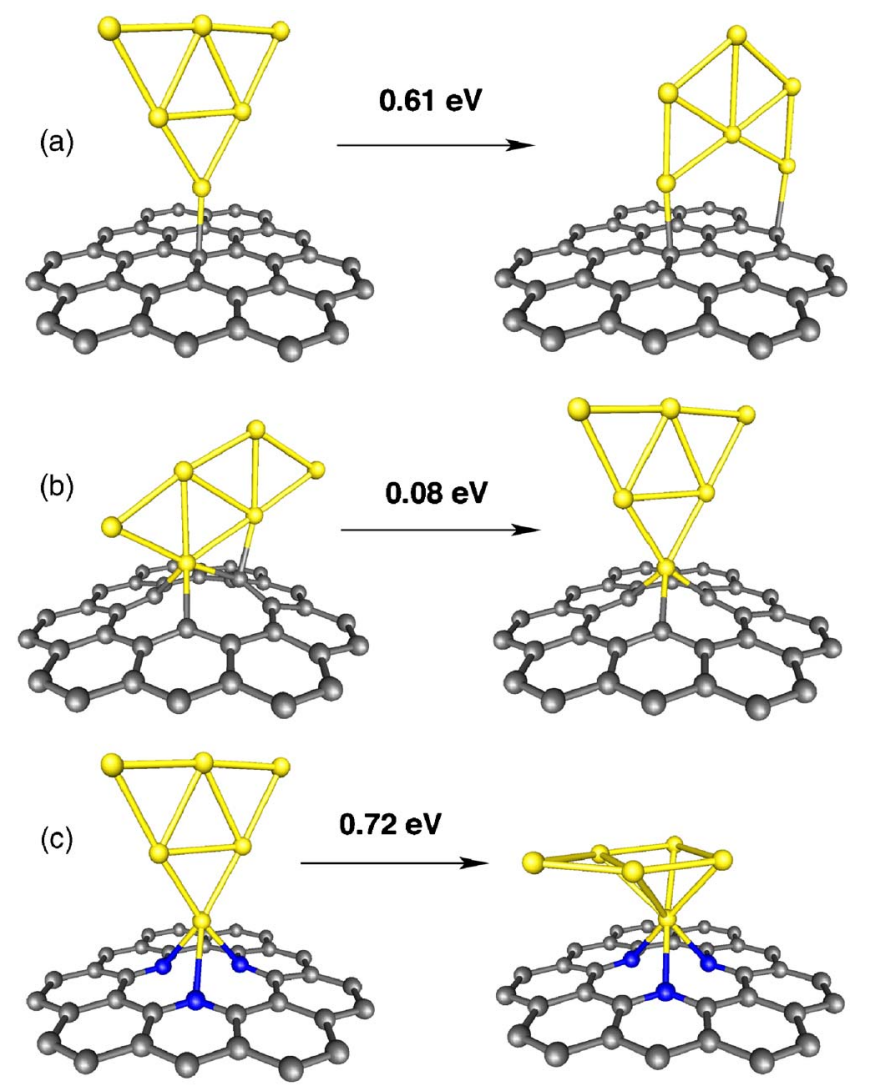

FIG. 5. (Color online) $\mathrm{Au}_{6}$ isomers on (a) perfect graphite (GR2), (b) surface vacancy (GR2-h), and (c) pyridinelike defect (GR2-N3). Each configuration includes the 37 (36) substrate atoms that are allowed to optimize. The left column shows the lowest energy configuration (see Table II).

with the defect. The $D_{3 h}$ edge orientation appears unstable as the cluster tilts, and the separation from the substrate is larger in the face-down orientation $(3.7 \AA)$ than with GR2. This is consistent with the experimental observation of hillocks in Ar sputtered HOPG samples, where the electron density protrudes farther at surface vacancies. ${ }^{45}$

The chemical stability of the pyridinelike defect GR2-N3 makes it more rigid against structural deformations. This can be seen as smaller $d_{\perp}(\mathrm{N})$ values in Table II. The cluster binding with defect is weaker than for GR2-h, but significantly stronger than for the perfect graphite surface. The energetic ordering is similar to GR2, and the $D_{3 h}$ configuration (apex) is the most stable. The energy difference with respect to the closest $C_{2 v}$ configuration is considerable $(\Delta E$ $=0.50 \mathrm{eV})$, and the first 3D isomer $C_{s}$ is $0.72 \mathrm{eV}$ higher in total energy. The largest vertical adsorption energies are observed for $D_{2 h}(1.37 \mathrm{eV})$ and $O_{h}$ configurations $(1.31 \mathrm{eV})$. For the $D_{3 h}$ edge and face-down orientations, the interaction with the defect is weak. In most cases the cluster is aligned along one of the substrate symmetry axes, and this causes symmetry breaking with respect to the three Au-N contacts. In the case of $D_{3 h}$ apex orientation, for example, one Au-N distance is shorter $(2.29 \AA)$ than the two others $(2.49 \AA)$. For $C_{5 v}$ this effect is hampered by the five-fold symmetry of the cluster, and all the Au-N distances are in the range 2.47$2.50 \AA$.
The charge density difference $(\Delta \rho)$ and electron localization function (ELF) of $\mathrm{Au}_{6}$ on GR2, GR2-h, and GR2-N3 are shown in Fig. 6. A polarization effect is seen for GR2 as the connecting $\mathrm{Au}$ atom donates charge (blue) to the $\mathrm{Au}-\mathrm{C}$ contact and nearest $\mathrm{Au}-\mathrm{Au}$ bonds (red). A slight accumulation of charge is also visible in the underlying graphene plane. For GR2-h, the effect is remarkably stronger due to the dangling bonds of $\mathrm{C}$ atoms that accept charge from the $\mathrm{Au}_{6}$ apex. This affects the $\mathrm{Au}_{6}$ polarization, and the $\mathrm{Au}$ atoms show an enhanced orbital dependent accumulation/depletion. $\mathrm{Au}_{6}$ on GR2-N3 displays similar features as on GR2 and GR2-h, but there is a charge depletion around the substrate $\mathrm{N}$ atoms.

The ELF reflects the probability of finding two electrons in the same location, and its range of values is between 0 (no localization, blue) and 1 (complete localization, red). A reference value of 0.5 corresponds to a homogeneous electron gas (metallic bonding), and can be seen as a green color within the $\mathrm{Au}_{6}$ cluster [Fig. 6(c)]. Similarly, the covalent $\mathrm{C}-\mathrm{C}$ bonds are red due to the electron pairing (see also the lone pair of nitrogen). For GR2 and GR2-N3, the clustersubstrate contact shows narrow necks of blue (delocalization) indicating no chemical bonding. The situation is different for GR2-h, as the dangling bonds (red) point towards $\mathrm{Au}$, and the ELF shows varying degrees of localization $(\geq 0.5)$ along the Au-C bond indicating a mixed metal-covalent character.

The laterally averaged charge density difference $\left(\Delta \rho_{\perp}\right)$ of $\mathrm{Au}_{6}$ on GR2 and GR2-h is shown in Fig. 7, where both the $\Gamma$ point and $5 \times 5 \times 1 \mathrm{k}$-point mesh results are included. As illustrated previously in Fig. 6 , the $\Delta \rho_{\perp}$ profiles show charge depletion in the vicinity of the substrate coordinating $\mathrm{Au}$, and the corresponding $\Delta q$ values are $-0.05 e$ and $-0.39 e$ for GR2 and GR2-h, respectively. On the other hand, the rest of $\mathrm{Au}_{6}$ tends to attract additional charge, and integration over the whole cluster $z$-range results in $\Delta q$ values of $0.13 e$ and $-0.06 e$ for GR2 and GR2-h (Table I), respectively. The two substrates show a qualitative difference in $\Delta \rho_{\perp}$ near the upper graphene layer, as GR2 has a negative node above the surface [see also Fig. 6(a)]. The oscillation of curves in the substrate range indicates that the lower graphene layer is also affected, and the largest deviations between the $\Gamma$-point approximation and the $5 \times 5 \times 1$ k-point mesh are observed there.

\section{CONCLUSION}

We have performed DFT calculations for the adsorption of $\mathrm{Au}$ adatoms and $\mathrm{Au}_{6}$ clusters on graphite (0001) surface with and without atomic defects. The simulations are performed with the scalar-relativistic Troullier-Martins pseudopotentials, an extensive plane wave basis set, and the GGA approximation for the exchange-correlation energy functional. The substrate is described by a periodic slab comprising two graphene layers, and this appears to be an adequate approximation for studying the interaction of gold particles with pointlike surface defects. In contrast to "cluster models," where the substrate is simulated as a large aromatic molecule with a significant energy gap between the occupied and unoccupied molecular orbitals (closed electron shell), 
GR2

(a)

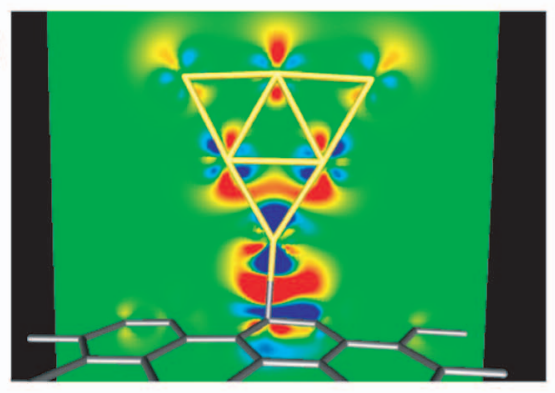

(b)

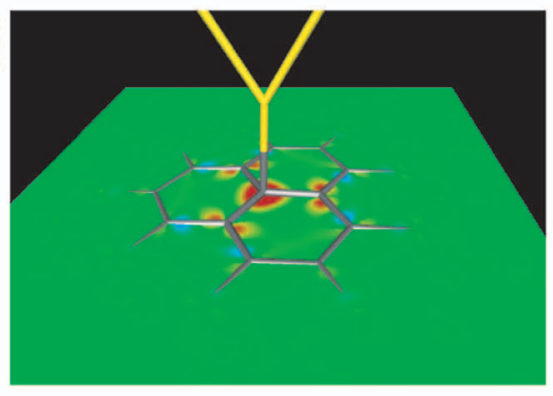

(c)

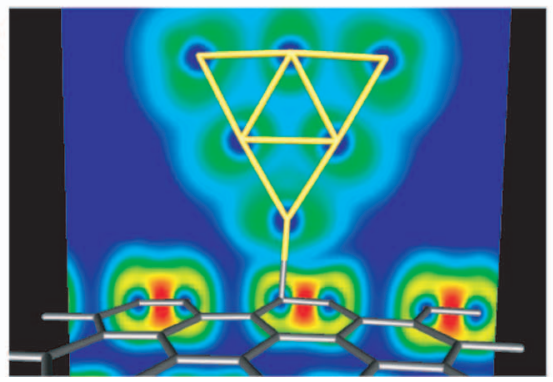

GR2-h
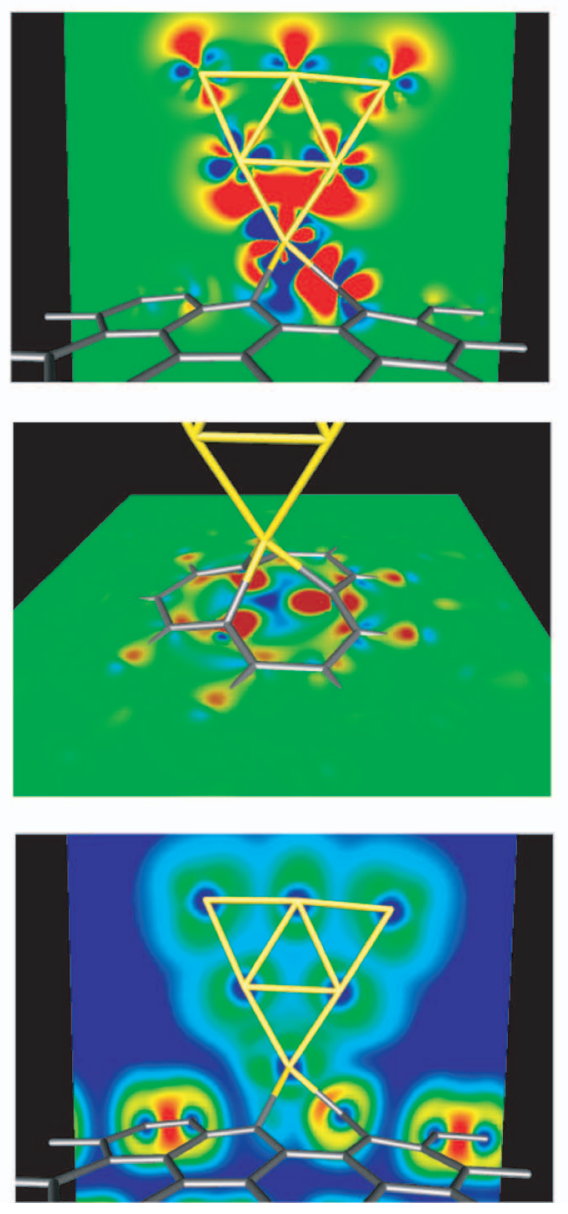

GR2-N3
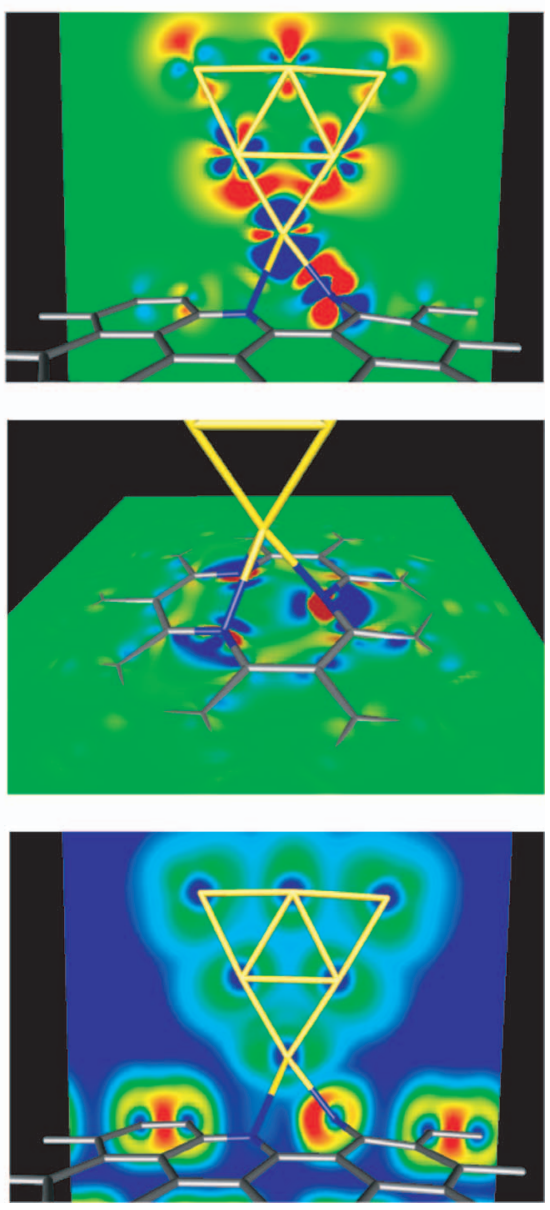

FIG. 6. (Color) Cutplane visualization of $\mathrm{Au}_{6}$ on HOPG. (a), (b) The charge density difference in $x z$ (cluster) and $x y$ planes (substrate), where the red color corresponds to accumulation $\left(0.0005 e / \AA^{3}\right.$ or more) and blue depletion $\left(-0.0005 e / \AA^{3}\right.$ or less). (c) The electron localization function (ELF) in $x z$ plane (cluster), where the red color corresponds to full localization (1.0, covalent bonds), green is analogous to homogeneous electron gas ( 0.5 , metallic bonding), and blue equals to low localization $(0.0)$.

the slab model enables incorporation of the electronic band structure of graphite (especially close to the Fermi energy). The large lateral dimension of the substrate makes it possible to use fewer $\mathbf{k}$ points, and the $\Gamma$-point approximation with a zero band gap for the chosen supercell is satisfactory for geometry optimization. The DFT method does not include the weak dispersion forces between the adsorbate and substrate, but this should not contribute significantly in the case of defects where the other types of cohesion dominate.

Our primary goal has been to study ways for improving gold adsorption on HOPG using pointlike defects, and the substrates considered show variation in their interaction with the gold particle (Table I). For the perfect graphite (0001) surface, our result for the Au adsorption energy $(0.3 \mathrm{eV})$ is slightly lower than the experimental value $E_{a}-E_{d}$ $=0.39 \pm 0.04 \mathrm{eV} \cdot{ }^{13,14} \mathrm{Au}_{6}$ adsorption is also weak $\left(E_{a}\right.$ $=0.3 \mathrm{eV}$ ). We note that semiempirical van der Waals corrections may change the energetics to favor the edge and facedown orientations of the $D_{3 h}$ isomer. In addition, the LDA calculations by Wang et al. for smaller Au clusters on HOPG (Refs. 20 and 21) show considerable adsorption energies for parallel orientations (surface separation $\sim 2.8 \AA$ ), and we presume that this would be the case also for the gold hexamer. Despite the small adsorption energies, substrate relaxation is observed both for $\mathrm{Au}$ and $\mathrm{Au}_{6}$ as the underlying $\mathrm{C}$ atoms are attracted towards the Au particle. The charge density difference plots show polarization effects in the adsorbate and HOPG, and the evaluated net charge transfer is 0.13-0.14 electrons towards gold. Experimentally, precipitation of $\mathrm{Au}$ nanoclusters on SWNT yields a corresponding $p$-doping effect in the nanotube. ${ }^{17}$

The strongest adsorption is found for a surface vacancy (GR2-h), where the dangling bonds of three $\mathrm{C}$ atoms form chemical bonds with $\mathrm{Au}$, and the corresponding binding energies are 2.4-2.6 eV. The vacancy exhibits a significant surface reconstruction, and the increased $\mathrm{Au}-\mathrm{C}$ coordination stabilizes the $D_{2 h}$ configuration of $\mathrm{Au}_{6}$. Other $\mathrm{Au}_{6}$ isomers are also closer in energy to the $D_{3 h}$ configuration (compared with perfect graphite), and the first 3D isomer is observed $0.27 \mathrm{eV}$ above the $D_{2 h}$ ground state. These results illustrate how deformations of the hexagonal graphite lattice increase the ability of the substrate to accommodate gold particles, and they agree with the experimental techniques where $\mathrm{Au}$ clusters either collide with HOPG with a high kinetic energy (pinning) ${ }^{24,25}$ or the clusters are trapped by preformed 


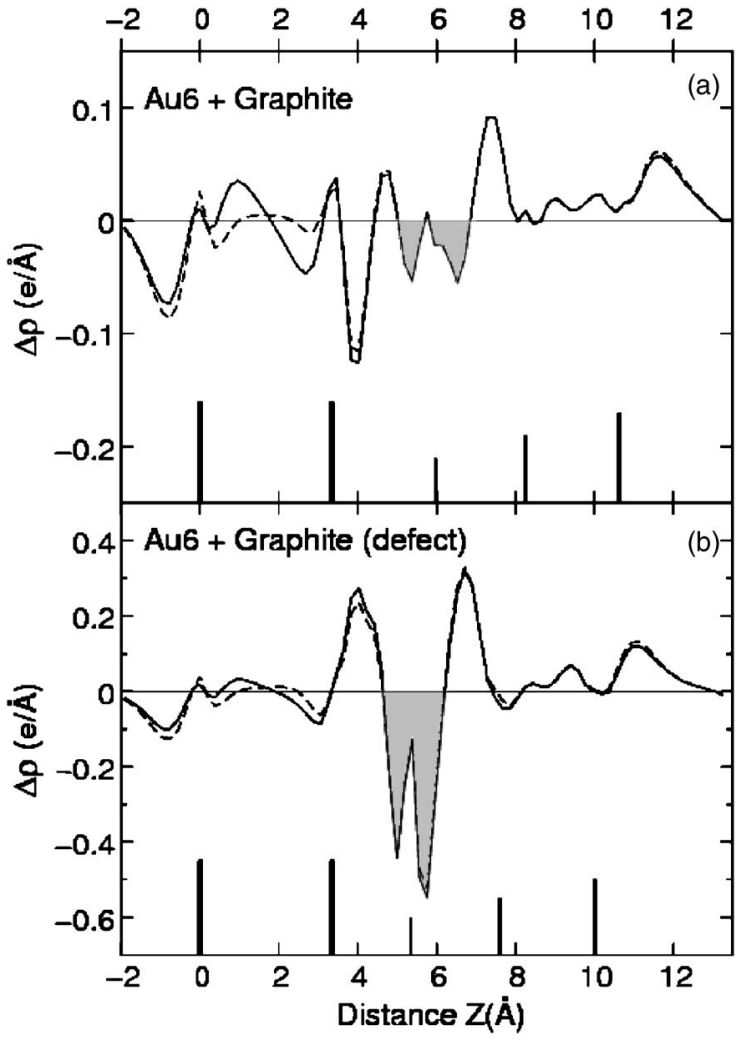

FIG. 7. Laterally averaged charge density difference $(\Delta \rho, z$ direction) of $\mathrm{Au}_{6}$ on (a) perfect graphite (GR2) and (b) surface vacancy (GR2-h). The solid and dashed lines correspond to the 5 $\times 5 \times 1$ k-point mesh and $\Gamma$-point approximation, respectively. The vertical bars denote the positions of graphene layers (thicker bars) and $\mathrm{Au}$ atoms (thinner bars). Notice the different $\Delta \rho$ scales.

nanometer-sized pits (oxidation of surface defects). ${ }^{26-28}$

Chemically stable pyridinelike defects provide another method for binding small Au particles. Our calculations (GR2-N3) result in the adsorption energies of 1.1-1.2 eV for $\mathrm{Au}$ and $\mathrm{Au}_{6}$, and the adsorbate-substrate interaction can be viewed as polarization $(\Delta \rho$, ELF). The substrate is rather rigid as the three $\mathrm{N}$ atoms remain in plane, and the clusters bind through corner atoms. The energy ordering of the different $\mathrm{Au}_{6}$ configurations resembles that of perfect graphite. Based on the experimental verification of their existence in $\mathrm{N}$-doped CNTs, ${ }^{31}$ we regard the pyridinelike defects as especially promising in the context of binding small gold particles on SWNTs.

The substitutional $\mathrm{B} / \mathrm{N}$ impurities appear less attractive for future applications. For boron, the adsorption energies are considerable, but they decrease from $\mathrm{Au}(1.2 \mathrm{eV})$ to $\mathrm{Au}_{6}$ $(0.8 \mathrm{eV})$. The charge density difference shows a complicated pattern as the "hole" in the substrate valence band interacts with the Au electron density, but there is no net charge transfer. In the case of graphitic nitrogen, the adsorption is weak $(0.2-0.6 \mathrm{eV})$ and is accompanied with a clear charge transfer as part of the additional conduction electron is donated to $\mathrm{Au} / \mathrm{Au}_{6}$.

Finally, we note that it should be possible to bind $\mathrm{Au}$ atoms and small Au clusters onto graphite (0001) and SWNT side walls by generating pointlike surface vacancies and pyridinelike defects. The applicability of these approaches is limited to low temperatures (small kinetic energy of diffusion) and small cluster sizes where the ratio of lowcoordinated (corner) $\mathrm{Au}$ atoms is high. For larger Au clusters, the collective binding through (111) facets presumably overcomes that of a single Au contact. The critical cluster size for the onset of this behavior remains open.

\section{ACKNOWLEDGMENTS}

Financial support from the Academy of Finland and from the European Community Project No. ULTRA-1D (NMP4CT-2003-505457) is acknowledged. The calculations were performed on IBM-SP4+ computers at the John von Neumann Institute for Computing (NIC), Forschungszentrum Jülich, Germany. We thank R. O. Jones for a critical reading of the manuscript.
${ }^{1}$ Physics and Chemistry of Finite Systems: From Clusters to Crystals I+II, edited by P. Jena, S. N. Khanna, and B. K. Rao (Kluwer, Dordrecht, 1992).

${ }^{2} \mathrm{H}$. Haberland, Clusters of Atoms and Molecules I+II (Springer, Berlin, 1994).

${ }^{3}$ K. H. Meiwes-Broer, Metal Clusters at Surfaces (Springer, Berlin, 2000).

${ }^{4}$ P. Jensen, Rev. Mod. Phys. 71, 1695 (1999).

${ }^{5}$ C. Binns, Surf. Sci. Rep. 44, 1 (2001).

${ }^{6}$ H. Haberland, Z. Insepov, and M. Moseler, Z. Phys. D: At., Mol. Clusters 26, 229 (1993).

${ }^{7}$ H. Häkkinen and M. Manninen, J. Chem. Phys. 105, 10565 (1996); H. Häkkinen and M. Manninen, Phys. Rev. Lett. 76, 1599 (1996); R. N. Barnett, H.-P. Cheng, H. Häkkinen, and U. Landman, J. Phys. Chem. 99, 7731 (1995).

${ }^{8}$ M. Moseler, H. Häkkinen, and U. Landman, Phys. Rev. Lett. 89, 176103 (2002).

${ }^{9}$ B. Huber, P. Koskinen, H. Häkkinen, and M. Moseler, Nat. Mater.
5, 44 (2006).

${ }^{10}$ H. Häkkinen, M. Moseler, and U. Landman, Phys. Rev. Lett. 89, 033401 (2002).

${ }^{11}$ S. Gilb, P. Weis, F. Furche, R. Ahlrichs, and M. M. Kappes, J. Chem. Phys. 116, 4094 (2002); F. Furche, R. Ahlrichs, P. Weis, C. Jacob, S. Gilb, T. Bierweiler, and M. M. Kappes, ibid. 117, 6982 (2002).

${ }^{12}$ H. Häkkinen, B. Yoon, U. Landman, X. Li, H.-J. Zhai, and L.-S. Wang, J. Phys. Chem. A 107, 6168 (2003).

${ }^{13}$ R. Anton and I. Schneidereit, Phys. Rev. B 58, 13874 (1998).

${ }^{14}$ R. Anton and P. Kreutzer, Phys. Rev. B 61, 16077 (2000).

${ }^{15}$ N. Vandamme, E. Janssens, F. Vanhouette, P. Lievens, and C. Van Haesendonck, J. Phys.: Condens. Matter 15, S2983 (2003).

${ }^{16}$ H. Dai, Surf. Sci. 500, 218 (2002).

${ }^{17}$ B. M. Quinn, C. Dekker, and S. G. Lemay, J. Am. Chem. Soc. 127, 6146 (2005).

${ }^{18}$ W. D. Luedtke and U. Landman, Phys. Rev. Lett. 82, 3835 (1999). 
${ }^{19}$ B. Yoon, W. D. Luedtke, and U. Landman, J. Phys. Chem. B 107, 5882 (2003).

${ }^{20}$ G. M. Wang, J. J. BelBruno, S. D. Kenny, and R. Smith, Phys. Rev. B 69, 195412 (2004).

${ }^{21}$ G. M. Wang, J. J. BelBruno, S. D. Kenny, and R. Smith, Surf. Sci. 576, 107 (2005)

${ }^{22}$ P. Jensen, X. Blase, and P. Ordejon, Surf. Sci. 564, 173 (2004).

${ }^{23}$ Q. Zhao, M. Buongiorno Nardelli, W. Lu, and J. Bernholc, Nano Lett. 5, 847 (2005).

${ }^{24}$ R. E. Palmer, S. Pratontep, and H.-G. Boyen, Nat. Mater. 2, 443 (2003).

${ }^{25}$ M. Di Vece, S. Palomba, and R. E. Palmer, Phys. Rev. B 72, 073407 (2005).

${ }^{26}$ H. Hövel, Th. Becker, A. Bettac, B. Reihl, M. Tschudy, and E. J. Williams, J. Appl. Phys. 81, 154 (1997).

${ }^{27}$ H. Hövel, Appl. Phys. A: Mater. Sci. Process. 72, 295 (2001).

${ }^{28}$ T. Irawan, I. Barke, and H. Hövel, Appl. Phys. A: Mater. Sci. Process. 80, 929 (2005).

${ }^{29}$ Y. Fan, B. R. Goldsmith, and P. G. Collins, Nat. Mater. 4, 906 (2005)

${ }^{30}$ A. Hashimoto, K. Suenaga, A. Gloter, K. Urita, and S. Iijima, Nature (London) 430, 870 (2004).

${ }^{31}$ R. Czerw, M. Terrones, J.-C. Charlier, X. Blase, B. Foley, R. Kamalakaran, N. Grobert, H. Terrones, D. Tekleab, P. M. Ajayan, W. Blau, M. Rühle, and D. L. Carroll, Nano Lett. 1, 457 (2001).

${ }^{32}$ See, for example, A. Savin, R. Nesper, S. Wengert, and T. F. Fässler, Angew. Chem., Int. Ed. Engl., 36, 1808 (1997).

${ }^{33}$ CPMD V3.9 Copyright IBM Corp 1990-2005, Copyright MPI für Festkörperforschung Stuttgart 1997-2001, www.cpmd.org

${ }^{34}$ J. P. Perdew, K. Burke, and M. Ernzerhof, Phys. Rev. Lett. 77, 3865 (1996).

${ }^{35}$ N. Troullier and J. L. Martins, Phys. Rev. B 43, 1993 (1991). For the carbon $2 s^{2} 2 p^{2}$ valence electrons, we use $s$-nonlocal and $p$-local components $\left(r_{\text {cut }}=1.23\right.$ a.u. $)$. The gold $5 d^{10} 6 s^{1}$ valence electrons experience angular components $s$ local, $p, d$, and $f$ nonlocal ( $r_{\text {cut }}=2.20,2.60,1.80$, and 1.60 a.u., respectively). Scalar-relativistic and nonlinear core corrections are included for $\mathrm{Au}$. The resulting $\mathrm{Au}_{2}$ bond length and dissociation energy are $2.526 \AA$ and $2.29 \mathrm{eV}$ compared to the experimental values $2.472 \AA$ and $2.29 \mathrm{eV}$ [J. Ho, K. M. Ervin, and W. C. Lineberger, J. Chem. Phys. 93, 6987 (1990)].

${ }^{36}$ A. Alavi, J. Kohanoff, M. Parrinello, and D. Frenkel, Phys. Rev. Lett. 73, 2599 (1994).

${ }^{37}$ K. Rytkönen, J. Akola, and M. Manninen, Phys. Rev. B 69, 205404 (2004)

${ }^{38}$ Test calculations for the Au adatom on a surface vacancy result in binding energies 2.33 and $2.38 \mathrm{eV}$ for two and three graphene layers, respectively. The interatomic distances are within $0.01 \AA$.

${ }^{39}$ K. R. Kganyago and P. E. Ngoepe, Phys. Rev. B 68, 205111 (2003).

${ }^{40}$ H. J. Monkhorst and J. D. Pack, Phys. Rev. B 13, 5188 (1976).

${ }^{41}$ R. Fletcher, Practical methods of Optimization (Wiley, New York, 1980), Vol. 1.

${ }^{42}$ The value of charge transfer depends on the method used for its determination. We use the method of laterally averaged charge density difference. See, for example, (a) F. Ancilotto and F. Toigo, Phys. Rev. B 47, 13713 (1993); (b) J. D. White, J. Cui, M. Strauss, R. D. Diehl, F. Ancilotto, and F. Toigo, Surf. Sci. 307-309, 1134 (1994); and (c) D. Lamoen and B. N. J. Persson, J. Chem. Phys. 108, 3332 (1998).

${ }^{43}$ P. O. Lehtinen, A. S. Foster, Y. Ma, A. V. Krasheninnikov, and R. M. Nieminen, Phys. Rev. Lett. 93, 187202 (2004).

${ }^{44}$ Y. Ma, P. O. Lehtinen, A. S. Foster, and R. M. Nieminen, New J. Phys. 6, 68 (2004).

${ }^{45}$ B. An, S. Fukuyama, and K. Yokogawa, J. Appl. Phys. 92, 2317 (2002). 